\title{
Risk factors associated with pulmonary hemorrhage and hemoptysis following percutaneous CT-guided transthoracic lung core needle biopsy: a retrospective study of 1,090 cases
}

\author{
Jianbin Zhu, Yaoming Qu, Xianlong Wang, Chunxiu Jiang, Jianhua Mo, Jiandong Xi, Zhibo Wen \\ Department of Radiology, Zhujiang Hospital, Southern Medical University, Guangzhou 510282, China
}

Correspondence to: Zhibo Wen. Department of Radiology, Zhujiang Hospital, Southern Medical University, Guangzhou 510282, China. Email: zhibowen@163.com.

\begin{abstract}
Background: Pulmonary hemorrhage and hemoptysis are the second-most common and potentially lifethreatening complications after pneumothorax following percutaneous computed tomography-guided transthoracic lung biopsy (PCTLB). Preventing hemorrhagic complications after PCTLB requires an accurate estimation of risk factors. This study investigated the risk factors associated with pulmonary hemorrhage and hemoptysis following PCTLB, and whether the ratio of main pulmonary artery diameter $(\mathrm{mPAD})$ to ascending aorta diameter (mPAD/AAD ratio) is a risk factor.
\end{abstract}

Methods: We retrospectively analyzed 1,090 cases of PCTLB obtained from 1,050 patients using a core needle. The risk factors for overall pulmonary hemorrhage, higher-grade pulmonary hemorrhage, and hemoptysis were evaluated by multivariate analysis of patient characteristics, computed tomography (CT) imaging data including pulmonary artery diameter (mPAD) to ascending aorta diameter (mPAD/AAD) ratio, technical variables related to the biopsy, and pathologic findings.

Results: Pulmonary hemorrhage occurred in 31.38\% (342/1,090) of PCTLB cases, including lower-grade $(24.4 \%, 266 / 1,090)$ and higher-grade hemorrhage $(6.97 \%, 76 / 1,090)$. The incidence of hemoptysis was $3.03 \%$ (33/1,090). Multivariate analysis revealed significant associations between overall pulmonary hemorrhage and lesion location in the lower lobe, subsolid and smaller lesions, greater lesion depth, and lung metastases. For higher-grade pulmonary hemorrhage, an mPAD/AAD ratio >1, smaller lesions, greater lesion depth, emphysema, and lung metastases were risk factors. Risk factors for hemoptysis were history of hypertension and lower- and higher-grade pulmonary hemorrhage.

Conclusions: Pulmonary artery enlargement detected by CT (mPAD/AAD ratio $>1$ ) is independently associated with higher-grade pulmonary hemorrhage following PCTLB.

Keywords: CT-guided lung biopsy; pulmonary hemorrhage; hemoptysis; pulmonary artery; risk analysis

Submitted Dec 07, 2019. Accepted for publication Apr 14, 2020.

doi: 10.21037/qims-19-1024

View this article at: http://dx.doi.org/10.21037/qims-19-1024

\section{Introduction}

Percutaneous computed tomography (CT)-guided transthoracic lung biopsy (PCTLB) is a minimally invasive technique and an important tool for the diagnosis of lung diseases, especially in cases in which the sputum cytology findings are negative, and the lesion cannot be reached in fiberoptic bronchoscopy (1-4). Although CT-guided lung biopsy is generally considered a safe procedure, it is nonetheless invasive and carries the risk of complications (5-7). Pulmonary hemorrhage and hemoptysis are the second most common and potentially life-threatening PCTLB-associated complications after pneumothorax $(3,8)$. Preventing hemorrhagic complications following PCTLB requires an accurate estimation of the risk factors. 
Pulmonary hypertension $(\mathrm{PH})$ has been considered a relative contraindication for PCTLB because it increases the risk of severe hemorrhagic complications $(2,9,10)$. Patients with suspected $\mathrm{PH}$ have a chest $\mathrm{CT}$ scan to allow evaluation of the lung parenchyma and pulmonary vasculature structures, such as pulmonary artery enlargement, which may result from $\mathrm{PH}$ (11-15). Prior studies have shown a moderate to a strong correlation between $\mathrm{PH}$ and pulmonary vascular measurements on CT images, including the ratio of the main pulmonary artery diameter to the ascending aorta diameter (mPAD/AAD ratio) at the level of the bifurcation of the main pulmonary artery (15-19). Although an enlarged pulmonary artery could be the result of several pathologic processes, including resting $\mathrm{PH}$, undiagnosed cardiovascular disease, or hyperinflation due to emphysema, $\mathrm{PH}$ is still the most common cause $(16,20,21)$. Furthermore, a recent study reported that enlarged mPAD observed by chest CT is not a risk factor for higher-grade pulmonary hemorrhage after PCTLB (22). In contrast, an extensive retrospective analysis concluded that $\mathrm{mPAD}$ enlargement was a significant risk factor for severe hemoptysis after CT-guided lung biopsy (23). Although several recent studies reported that the pulmonary arterial hypertension did not increase the risk of hemorrhagic complication after PCTLB $(22,24)$, these studies were limited by small sample size and included patients with less severe $\mathrm{PH}$, and there is thus insufficient evidence to establish a clear relationship between $\mathrm{PH}$ and hemorrhagic complications. To our knowledge, no prior studies have used $\mathrm{mPAD} / \mathrm{AAD}$ ratio based on $\mathrm{CT}$ to determine whether there is an increased risk of pulmonary hemorrhage and hemoptysis after PCTLB.

Thus, the purpose of our study was to evaluate the related risk factors for pulmonary hemorrhage and hemoptysis following PCTLB, focusing on whether mPAD/ $\mathrm{AAD}$ ratio is associated with hemorrhagic complications.

\section{Methods}

\section{Study population}

We retrospectively collected electronic medical records (EMRs) of 1,122 cases from 1,082 patients diagnosed with lung disease who underwent PCTLB in the radiology department of our hospital from January 2014 to September 2017. Inclusion criteria were patients for whom images (including preoperative chest CT images for measuring mPAD and AAD values) and clinical information were available. Furthermore, those CT-guided lung biopsies performed without core needles were excluded. We excluded 32 PCTLB cases according to these criteria. Ultimately, 1,090 PCTLB procedures in 1,050 patients (732 men and 318 women; mean age, 58.09 years; range, $13-88$ years) were included in the analysis. There were 37 patients who underwent repeated biopsies (34 patients with 2 biopsies and 3 patients with 3 biopsies), and these were considered as separate procedures in the calculations as different variables were examined for each biopsy. The study protocol was approved by our institutional review board (2019-KY-056-01) with a waiver of informed consent obtained from each patient.

\section{Biopsy procedure}

All PCTLBs were performed under CT guidance. Patients underwent PCTLB (Brilliance 16; Philips, Amsterdam, The Netherlands) with the following CT scan parameters: helical scan; tube voltage, $120 \mathrm{kV}$; tube current, $80 \mathrm{~mA}$; slice thickness, $5 \mathrm{~mm}$; and CT interval, $5 \mathrm{~mm}$. Core needle biopsies were performed using an 18G-100/150 mm needle (OptiMed Medical Instruments, Ettlingen, Germany; cat. no. 1399-1210) by or under the supervision of a chest radiologist (Z. Wen., with 28 years of experience in PCTLB). Bleeding profile, including platelet count, clotting time, prothrombin time, and activated partial thromboplastin time, was controlled, and anticoagulants were withheld for $>1$ week before the biopsy. Electrocardiogram and pulmonary function tests were not required by our biopsy protocol. Drugs and equipment for potential complications were prepared beforehand.

All patients first underwent a preoperatively planned chest CT scan before PCTLB. The images were reviewed by radiologists, who assessed the location and size of the lesion and determined the optimal position of the patient (prone, supine, or lateral decubitus) for the biopsy by drawing the needle path, marking the biopsy spot on patient's body surface, noting the biopsy parameter, and selecting the length of the core needle (100 or $150 \mathrm{~mm}$ ). The procedure involved passing the biopsy needle through the skin, chest wall, pleura, and lesion to minimize damage to lung tissues, while avoiding ribs, vessels, interlobar fissures, and intercostal nerves. After local infiltration anesthesia, the needle was inserted at the optimal angle until the tip reached the edge of the lesion, which was determined by several small-scale CT scans during the procedure. The lesion was cut once or more time to obtain tissue samples 

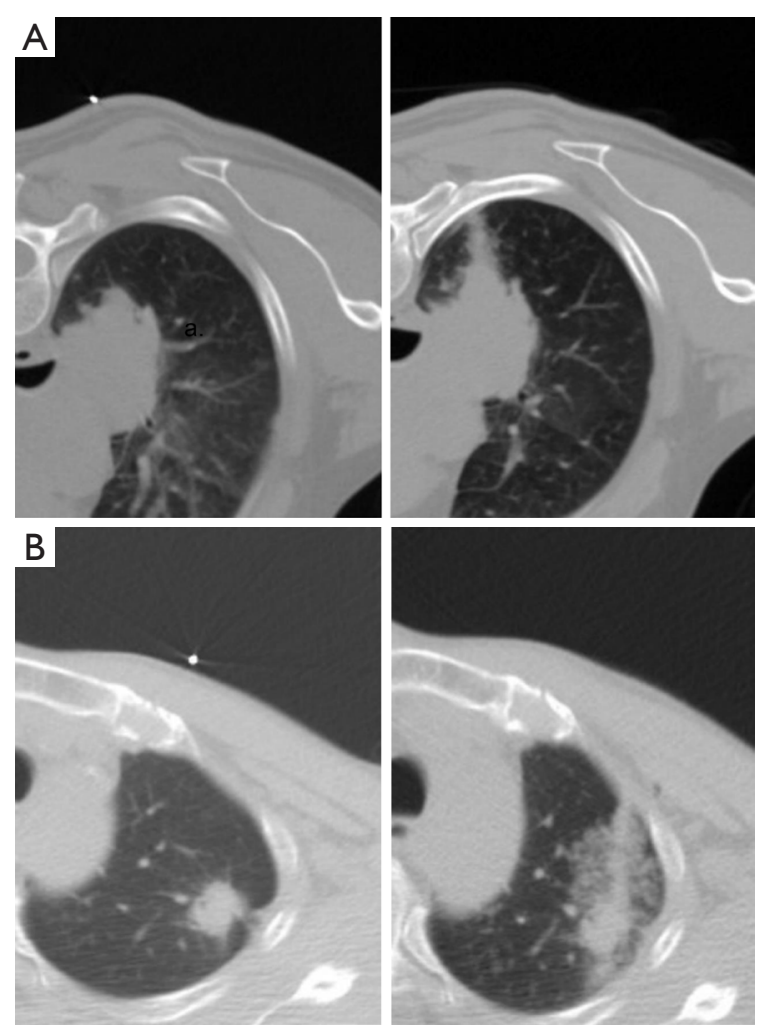

Figure 1 Pulmonary hemorrhage grading scheme was shown on chest CT scans. (A) Lowergrade pulmonary hemorrhage in 61 years old man. There is new ground-glass and consolidative opacity along needle tract measuring $2 \mathrm{~cm}$ or less in width after CT-guided lung core needle biopsy of right lower lobe solid lesion; (B) higher-grade pulmonary hemorrhage in 59 years old man. After CT-guided lung biopsy of left upper lobe solid nodule, there is a larger area of new ground-glass and consolidative opacity measuring more than $2 \mathrm{~cm}$ around the needle.

which were then fixed with $10 \%$ formaldehyde and sent to the laboratory for pathology examination. After the biopsy, the patient was ordered to rest in the supine position, and a control CT scan was routinely performed to determine whether there were immediate PCTLB-related complications.

\section{Definition and choice of study variables}

Two experienced thoracic radiologists (Zhibo Wen and Xianlong Wang) and two research fellows (Jianbin Zhu and Yaoming Qu) evaluated all cases in the image database of our institution, and reviewed institutional medical records including the relevant diagnostic, imaging, and pathology examination results.

Pulmonary hemorrhage was defined as new hyperdense consolidation or ground-glass opacity in post-biopsy images. The severity of pulmonary hemorrhage was categorized according to a previous study (22) but simplified from the previous hemorrhage grading scheme for the sake of convenience. The grading was as follows: no hemorrhage; lower-grade pulmonary hemorrhage $=$ needle tract hemorrhage $\leq 2 \mathrm{~cm}$ in width; and higher-grade pulmonary hemorrhage $=$ larger area of hemorrhage $>2 \mathrm{~cm}$ around the needle encompassing the entire lobar and hemothorax. The area of pulmonary hemorrhage was measured from CT images (Figure 1).

The occurrence and severity of hemoptysis during or after PCTLB were recorded based on EMRs of our hospital in one week observation. Any expectoration of bloodstreaked sputum, blood, or fresh blood after the procedure was considered as hemoptysis. Hemoptysis occurring in the CT scanning room and ward was documented by the clinician and nurse, respectively, and was usually categorized as mild-to-moderate (minor, self-limiting or treatable) or massive/severe (if the volume of blood was large enough to be life-threatening), according to the severity of the manifestation (25).

\section{Clinical data}

The clinical information obtained from the EMR of each patient included age; sex; the history of chronic obstructive pulmonary disease, hypertension, or diabetes mellitus; and prior malignancy.

\section{CT images}

The mPAD at the level of its bifurcation and the maximum dimension of AAD from the same axial chest CT (plain or contrast-enhanced CT) image were measured as previously described (Figure 2) (18,26). An enlarged mPAD was defined as $\geq 2.95 \mathrm{~cm}(12,22,23)$. A threshold of mPAD/AAD ratio $>1$ was defined as indicating the presence of relative pulmonary artery enlargement, and this has been used previously for accurately diagnosing $\mathrm{PH}$ owing to its highly balanced diagnostic ability $(12,27)$.

\section{Lesion location}

Pulmonary lesions were classified according to their lobar location: left upper lobe, left lower lobe, left hilar, right upper lobe, right middle lobe, right lower lobe, or right hilar. 


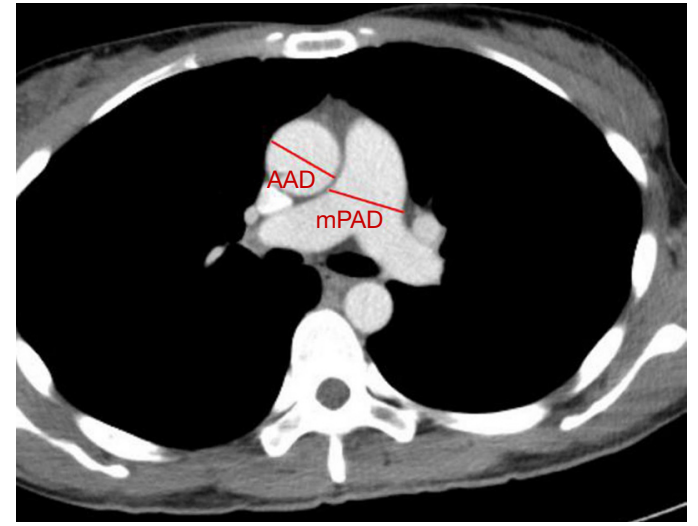

Figure 2 Measurement of main pulmonary artery diameter $(\mathrm{mPAD})$ and ascending aorta diameter (AAD). Axial chest contrastenhanced CT image: measurement of the MPAD and the AAD by selecting the widest diameter at the level of the main pulmonary artery bifurcation, were used to calculate the mPAD/AAD ratio.

\section{Lesion characteristics}

Pulmonary lesions were classified as solid (including solid nodules or masses), subsolid $(22,28)$ (including ground-glass and partly solid), consolidation (appearing as a homogeneous increase in pulmonary parenchymal attenuation that obscures the margins of vessels and airway walls), or cavitary.

\section{Lesion size}

Lesion size was based on the measurement along the maximum long-axis diameter, and was classified into small size (diameter $\leq 3 \mathrm{~cm}$ ) or large size (diameter $>3 \mathrm{~cm}$ ).

\section{Lesion depth}

Lesion depth was measured from the chest wall to the nearest edge of the lesion along the needle path and was categorized as subpleural and superficial location (distance $\leq 3 \mathrm{~cm}$ ), intermediate location (distance $>3$ and $\leq 5 \mathrm{~cm}$ ), or deep location (distance $>5 \mathrm{~cm}$ ).

\section{Emphysema}

The presence of emphysema in the lung parenchyma surrounding the target lesion was assessed as absent or present.

\section{Technique-related variables}

Technique-related variables included the number of needle passes (given that lesions were cut one or more time to obtain tissue samples), needle angle (measured as the angle between line drawn along the needle trajectory and tangential to the pleura, with the tangent of the right part as the baseline, and categorized as $<90^{\circ}$ or $\geq 90^{\circ}$ ), and location of biopsy (anterior chest wall, lateral chest wall, or posterior chest wall, which was determined relative to the axillary front line and axillary posterior line).

\section{Pathological results}

Based on a review of the pathology reports, biopsy specimens were categorized as tumor (lung adenocarcinoma, lung squamous cell carcinoma, small cell lung cancer, lung metastases, or other tumors types), nontumor (inflammatory changes, tuberculosis, mycotic infection, or other nontumors), or non-diagnostic.

\section{Intervention}

Serious complications requiring surgical intervention, especially in cases where general complications developed continuously and were not controlled by drugs-included uncontrolled hemoptysis, unconsciousness, asphyxia, and coma, and were handled by various emergency measures.

\section{Statistical analysis}

Mean differences between two groups were evaluated by univariate analysis (chi-squared test and Fisher's exact test for categorical variables and Student's $t$-test for continuous variables). Binary logistic regression analysis was performed to find potential risk factors for overall pulmonary hemorrhage, higher-grade pulmonary hemorrhage, and hemoptysis. Variables with $\mathrm{P}$ values $<0.1$ in the univariate analysis were included in stepwise forward multivariate logistic models to adjust for confounders. Risk factors for patients are presented as odds ratios (OR) with a $95 \%$ confidence interval (CI). SPSS version 21.0 for Windows (IBM, Armonk, NY, USA) was used for all statistical analyses, and a $\mathrm{P}$ value $<0.05$ was considered statistically significant.

\section{Results}

\section{Overall outcomes}

Pulmonary hemorrhage occurred in $342 / 1,090$ cases (31.38\%), 266/1,090 PCTLBs (24.40\%) showed lowergrade pulmonary hemorrhage, and $76(6.97 \%)$ showed higher-grade pulmonary hemorrhage, with no cases of 
hemothorax. Hemoptysis occurred in 33/1,090 cases $(3.03 \%)$. Mild-to-moderate hemoptysis occurred in $28 / 1,090$ cases $(2.6 \%)$, and massive or severe hemoptysis occurred in 5/1,090 cases ( $0.4 \%)$. Overall mean mPAD and AAD were $2.97 \pm 0.49$ and $3.39 \pm 0.44 \mathrm{~cm}$, respectively, and the $\mathrm{mPAD} / \mathrm{AAD}$ ratio was $0.88 \pm 0.15$.

\section{Univariate analysis}

\section{Pulmonary hemorrhage}

We compared clinical and radiological data between patients with and without pulmonary hemorrhage (Table 1). Pulmonary hemorrhage was more likely to occur in patients with prior malignancy $(\mathrm{P}=0.002)$, in the right hilar vs. non-right-hilar regions $(\mathrm{P}<0.001)$, and in subsolid vs. solid, consolidation, and cavitary lesions $(\mathrm{P}=0.003)$; and was significantly less likely to occur with consolidation or cavitary lesions $v s$. other lesion types (solid and subsolid) $(\mathrm{P}<0.001)$. Pulmonary hemorrhage was also more likely to occur when lesions were small-sized $(\mathrm{P}<0.001)$ and had a deep location $(\mathrm{P}<0.001)$, surrounding emphysema $(\mathrm{P}=0.012)$, and lung metastases $(\mathrm{P}<0.001)$.

No or lower-grade pulmonary hemorrhage occurred in 1,014 of the 1,090 cases (93.03\%). Univariate analysis showed that higher-grade hemorrhage was more likely to occur when there was a prior malignancy $(\mathrm{P}=0.028)$, an $\mathrm{mPAD} / \mathrm{AAD}$ ratio $>1(\mathrm{P}=0.037)$, or when lesions were smallsized $(\mathrm{P}<0.001)$, had a deep location $(\mathrm{P}<0.001)$, surrounding emphysema $(\mathrm{P}<0.001)$, lung metastases $(\mathrm{P}=0.001)$, or located in the posterior chest wall $(\mathrm{P}=0.016)$ (Table 2). Higher-grade pulmonary hemorrhage was unrelated to $\mathrm{mPAD}, \mathrm{AAD}$, or other variables.

\section{Hemoptysis}

Hemoptysis was more likely to occur with a history of hypertension $(\mathrm{P}=0.011)$ and with deeper lesions $(\mathrm{P}=0.026)$ (Table 3). In 22/33 cases, hemoptysis was accompanied by pulmonary hemorrhage $(\mathrm{P}<0.001)$, which was more likely to be of a higher grade $(\mathrm{P}<0.001)$. Notably, all patients with massive or severe hemoptysis had a higher-grade pulmonary hemorrhage.

\section{Multivariate analysis}

To further clarify the association between clinicoradiologic characteristics and hemorrhagic complications, we used overall pulmonary hemorrhage, higher-grade pulmonary hemorrhage, and hemoptysis as dependent variables for logistic regression modeling (Table 4). Left lower lobe $(\mathrm{P}=0.006, \mathrm{OR}=1.948,95 \% \mathrm{CI}: 1.21-3.14)$, right lower lobe ( $\mathrm{P}=0.013$, OR $=1.754,95 \%$ CI: $1.125-2.734)$, or right hilar $(\mathrm{P}=0.009, \mathrm{OR}=5.368,95 \% \mathrm{CI}: 1.518-18.986)$ location; subsolid $(\mathrm{P}=0.007$, OR $=3.468,95 \% \mathrm{CI}: 1.405-8.561)$, solid ( $\mathrm{P}=0.016$, OR $=2.618,95 \%$ CI: 1.196-5.728), or small $(\mathrm{P}=0.003, \mathrm{OR}=1.628,95 \%$ CI: 1.186-2.236) lesions, lesions with intermediate $(\mathrm{P}<0.001$, OR $=4.558,95 \%$ CI: $2.141-$ 9.704) or deep $(\mathrm{P}<0.001$, OR $=25.641,95 \%$ CI: $12.276-$ 53.56) location; and lung metastases $(\mathrm{P}<0.001, \mathrm{OR}=6.695$, 95\% CI: 2.618-17.122) were significant independent risk factors of overall pulmonary hemorrhage.

For higher-grade pulmonary hemorrhage, mPAD/AAD ratio $>1$ ( $\mathrm{P}=0.03, \mathrm{OR}=1.871,95 \% \mathrm{CI}: 1.063-3.294)$, lesions that were small $(\mathrm{P}=0.023, \mathrm{OR}=1.769,95 \% \mathrm{CI}: 1.081-2.897)$ or had a deep location $(\mathrm{P}=0.001$, OR $=5.88,95 \% \mathrm{CI}$ : 2.046-16.898) or surrounding emphysema $(\mathrm{P}<0.001$, OR $=2.81,95 \%$ CI: $1.709-4.621)$; and lung metastases $(\mathrm{P}<0.001$, OR $=6.687,95 \%$ CI: 2.629-17.011) were significant independent risk factors in the multivariate analysis.

Significant risk factors for hemoptysis were a history of hypertension $(\mathrm{P}=0.016$, OR $=2.507,95 \%$ CI: $1.191-$ 5.277), lower-grade pulmonary hemorrhage $(\mathrm{P}=0.008$, $\mathrm{OR}=3.065,95 \% \mathrm{CI}: 1.332-7.053)$, and higher - grade pulmonary hemorrhage $(\mathrm{P}<0.001, \mathrm{OR}=10.39,95 \% \mathrm{CI}$ : 4.226-25.541).

\section{Intervention}

Pulmonary hemorrhage that required intervention occurred in $6 / 1,090$ cases $(0.5 \%)$, of which 5 had hemoptysis. One patient who died $(0.09 \%)$ had a small lesion with a diameter of $1.97 \mathrm{~cm}$ and a small amount of hemoptysis after PCTLB, after developing sudden coma and experiencing cardiac arrest. The patient was sent to the emergency department for resuscitation; this was unsuccessful, and the patient remained in a deep coma and eventually died. Three patients with massive hemoptysis accompanied by blood oxygen saturation lost consciousness and were admitted for endotracheal intubation and emergency thoracotomy. There was 1 case in which CT images showed mild pulmonary hemorrhage and slight pneumothorax after the procedure, with the patient losing consciousness. One patient with higher-grade pulmonary hemorrhage, and no hemoptysis case was admitted for surgery because of unconsciousness. These five patients were stabilized by prompt intervention. 
Table 1 Results of univariate analysis to determine risk factors for pulmonary hemorrhage following percutaneous CT-guided transthoracic lung biopsy

\begin{tabular}{|c|c|c|c|}
\hline Variable & $\begin{array}{l}\text { No pulmonary } \\
\text { hemorrhage } \\
\quad(n=748)\end{array}$ & $\begin{array}{l}\text { Pulmonary } \\
\text { hemorrhage } \\
(n=342)\end{array}$ & $P$ value \\
\hline Age $(y)$ & $58.0 \pm 13.1$ & $57.8 \pm 12.5$ & $0.851^{a}$ \\
\hline Sex & & & $0.085^{b}$ \\
\hline Male & 535 (71.5) & 227 (66.4) & \\
\hline Female & $213(28.5)$ & $115(33.6)$ & \\
\hline $\begin{array}{l}\text { History of chronic } \\
\text { obstructive } \\
\text { pulmonary disease }\end{array}$ & $50(6.7)$ & $19(5.6)$ & $0.478^{b}$ \\
\hline $\begin{array}{l}\text { History of } \\
\text { hypertension }\end{array}$ & $140(18.7)$ & $70(9.4)$ & $0.496^{b}$ \\
\hline $\begin{array}{l}\text { History of diabetes } \\
\text { mellitus }\end{array}$ & $99(13.2)$ & $31(9.1)$ & $0.055^{\mathrm{b}}$ \\
\hline Prior malignancy & $34(4.5)$ & $32(9.4)$ & $0.002^{b}$ \\
\hline mPAD & & & $0.062^{b}$ \\
\hline$<2.95 \mathrm{~cm}$ & 381 (50.9) & $195(57.0)$ & \\
\hline$\geq 2.95 \mathrm{~cm}$ & 367 (49.1) & $147(43.0)$ & \\
\hline $\mathrm{AAD}(\mathrm{cm})$ & $3.38 \pm 0.45$ & $3.40 \pm 0.43$ & $0.429^{a}$ \\
\hline mPAD/AAD ratio & & & $0.125^{b}$ \\
\hline$\leq 1$ & 608 (81.3) & 291 (85.1) & \\
\hline$>1$ & $140(18.7)$ & $51(14.9)$ & \\
\hline Lesion location & & & $0.001^{\mathrm{b}}$ \\
\hline Left upper lobe & 224 (29.9) & $79(23.1)$ & \\
\hline Left lower lobe & $130(17.4)$ & $56(16.4)$ & \\
\hline Left hilar & $6(0.8)$ & $7(2.0)$ & \\
\hline Right upper lobe & 217 (29.0) & 107 (31.3) & \\
\hline Right middle lobe & $30(4.0)$ & $13(3.8)$ & \\
\hline Right lower lobe & 137 (18.3) & 68 (19.9) & \\
\hline Right hilar & $4(0.5)$ & $12(3.5)$ & \\
\hline $\begin{array}{l}\text { Lesion } \\
\text { characteristics }\end{array}$ & & & $<0.001^{\mathrm{b}}$ \\
\hline Solid & 585 (78.2) & 287 (83.9) & \\
\hline Subsolid & $50(6.7)$ & $41(12.0)$ & \\
\hline Consolidation & $70(9.4)$ & $5(1.5)$ & \\
\hline Cavitary & $43(5.7)$ & $9(2.6)$ & \\
\hline Lesion size & & & $<0.001^{\mathrm{b}}$ \\
\hline$\leq 3 \mathrm{~cm}$ & 225 (30.1) & $160(46.8)$ & \\
\hline$>3 \mathrm{~cm}$ & $523(69.9)$ & $182(53.2)$ & \\
\hline
\end{tabular}

Table 1 (continued)
Table 1 (continued)

\begin{tabular}{|c|c|c|c|}
\hline Variable & $\begin{array}{l}\text { No pulmonary } \\
\text { hemorrhage } \\
\quad(n=748)\end{array}$ & $\begin{array}{l}\text { Pulmonary } \\
\text { hemorrhage } \\
(n=342)\end{array}$ & $P$ value \\
\hline Lesion depth & & & $<0.001^{b}$ \\
\hline$\leq 3 \mathrm{~cm}$ & $188(25.1)$ & $9(2.6)$ & \\
\hline $3-5 \mathrm{~cm}$ & $318(42.5)$ & $66(19.3)$ & \\
\hline$>5 \mathrm{~cm}$ & 242 (32.4) & 267 (78.1) & \\
\hline Emphysema & $166(22.2)$ & $100(29.2)$ & $0.012^{b}$ \\
\hline $\begin{array}{l}\text { Number of needle } \\
\text { passes }\end{array}$ & & & $0.103^{b}$ \\
\hline One & $136(18.2)$ & $79(23.1)$ & \\
\hline Two & $599(80.1)$ & $260(76.0)$ & \\
\hline Three or more & $13(1.7)$ & $3(0.9)$ & \\
\hline Needle angle & & & $0.415^{\mathrm{b}}$ \\
\hline$<90^{\circ}$ & $442(59.1)$ & $211(61.7)$ & \\
\hline$\geq 90^{\circ}$ & $306(40.9)$ & $131(38.3)$ & \\
\hline Location of biopsy & & & $0.18^{b}$ \\
\hline $\begin{array}{l}\text { Anterior chest } \\
\text { wall }\end{array}$ & $258(34.5)$ & $116(33.9)$ & \\
\hline Lateral chest wall & 166 (22.2) & $61(17.8)$ & \\
\hline $\begin{array}{l}\text { Posterior chest } \\
\text { wall }\end{array}$ & $324(43.3)$ & 165 (48.2) & \\
\hline Pathological results & & & $<0.001^{b}$ \\
\hline $\begin{array}{l}\text { Lung } \\
\text { adenocarcinoma }\end{array}$ & $275(36.8)$ & $148(43.3)$ & \\
\hline $\begin{array}{l}\text { Lung squamous } \\
\text { cell carcinoma }\end{array}$ & $80(10.7)$ & $19(5.6)$ & \\
\hline $\begin{array}{l}\text { Small cell lung } \\
\text { cancer }\end{array}$ & $31(4.1)$ & $21(6.1)$ & \\
\hline Lung metastases & $12(1.6)$ & $19(5.6)$ & \\
\hline Other tumors & $51(6.8)$ & $17(5.0)$ & \\
\hline $\begin{array}{l}\text { Inflammatory } \\
\text { changes }\end{array}$ & 149 (19.9) & $52(15.2)$ & \\
\hline Tuberculosis & $49(6.6)$ & $23(6.7)$ & \\
\hline Mycotic infection & $23(3.1)$ & $6(1.8)$ & \\
\hline Other non-tumors & $42(5.6)$ & $16(4.7)$ & \\
\hline Non-diagnostic & $36(4.8)$ & $21(6.1)$ & \\
\hline
\end{tabular}

Pulmonary hemorrhage was significantly more likely to occur with a right hilar location $(P<0.001)$, subsolid lesions $(P=0.003)$, and lung metastases $(P<0.001)$. Continuous variables are expressed as mean \pm SD. Categorical data are No. (\%) of patients undergoing percutaneous CT-guided transthoracic lung biopsy. a , Student's $t$-test; ${ }^{\text {b }}$, Chi-square test. mPAD, main pulmonary artery diameter; AAD, ascending aorta diameter. 
Table 2 Results of univariate analysis to determine risk factors for higher-grade pulmonary hemorrhage following percutaneous CTguided transthoracic lung biopsy

\begin{tabular}{|c|c|c|c|}
\hline Variable & $\begin{array}{c}\text { No/lower-grade } \\
\text { hemorrhage } \\
(n=1,014)\end{array}$ & $\begin{array}{l}\text { Higher-grade } \\
\text { hemorrhage } \\
\quad(n=76)\end{array}$ & $P$ value \\
\hline Age (y) & $57.9 \pm 12.9$ & $57.7 \pm 11.6$ & $0.864^{a}$ \\
\hline Sex & & & $0.973^{\mathrm{b}}$ \\
\hline Male & 709 (69.9) & $53(69.7)$ & \\
\hline Female & $305(30.1)$ & $23(30.3)$ & \\
\hline $\begin{array}{l}\text { History of chronic } \\
\text { obstructive } \\
\text { pulmonary disease }\end{array}$ & $64(6.6)$ & $5(6.6)$ & $0.993^{b}$ \\
\hline $\begin{array}{l}\text { History of } \\
\text { hypertension }\end{array}$ & 196 (19.3) & $14(18.4)$ & $0.846^{b}$ \\
\hline $\begin{array}{l}\text { History of diabetes } \\
\text { mellitus }\end{array}$ & $124(12.2)$ & $6(7.9)$ & $0.261^{\mathrm{b}}$ \\
\hline Prior malignancy & $57(5.6)$ & $9(11.8)$ & $0.028^{\mathrm{b}}$ \\
\hline mPAD & & & $0.321^{b}$ \\
\hline$<2.95 \mathrm{~cm}$ & $540(59.8)$ & $36(47.4)$ & \\
\hline$\geq 2.95 \mathrm{~cm}$ & $474(40.2)$ & $40(52.6)$ & \\
\hline AAD $(\mathrm{cm})$ & $3.38 \pm 0.44$ & $3.44 \pm 0.45$ & $0.275^{\mathrm{a}}$ \\
\hline mPAD/AAD ratio & & & $0.037^{b}$ \\
\hline$\leq 1$ & $843(83.1)$ & $56(73.7)$ & \\
\hline$>1$ & $171(16.9)$ & $20(26.3)$ & \\
\hline Lesion location & & & $0.162^{b}$ \\
\hline $\begin{array}{l}\text { Upper and middle } \\
\text { lobes }\end{array}$ & $629(62.0)$ & $41(53.9)$ & \\
\hline $\begin{array}{l}\text { Lower lobe and } \\
\text { lung hilar }\end{array}$ & $385(38.0)$ & $35(46.1)$ & \\
\hline Lesion characteristics & & & $0.126^{\mathrm{b}}$ \\
\hline Solid & 809 (79.8) & $63(82.9)$ & \\
\hline Subsolid & $82(8.1)$ & $9(11.8)$ & \\
\hline $\begin{array}{l}\text { Consolidation or } \\
\text { cavitary }\end{array}$ & $123(12.1)$ & $4(5.3)$ & \\
\hline Lesion size & & & $<0.001^{b}$ \\
\hline$\leq 3 \mathrm{~cm}$ & $344(33.9)$ & $43(56.6)$ & \\
\hline$>3 \mathrm{~cm}$ & $670(66.1)$ & $33(43.4)$ & \\
\hline Lesion depth & & & $<0.001^{\mathrm{b}}$ \\
\hline$\leq 3 \mathrm{~cm}$ & $193(19.0)$ & $4(5.3)$ & \\
\hline
\end{tabular}

Table 2 (continued)
Table 2 (continued)

\begin{tabular}{|c|c|c|c|}
\hline Variable & $\begin{array}{c}\text { No/lower-grade } \\
\text { hemorrhage } \\
(n=1,014)\end{array}$ & $\begin{array}{c}\text { Higher-grade } \\
\text { hemorrhage } \\
\quad(n=76)\end{array}$ & $P$ value \\
\hline $3-5 \mathrm{~cm}$ & $372(36.7)$ & $12(15.8)$ & \\
\hline$>5 \mathrm{~cm}$ & $449(44.3)$ & $60(78.9)$ & \\
\hline Emphysema & $231(22.8)$ & $35(46.1)$ & $<0.001^{\mathrm{b}}$ \\
\hline \multicolumn{2}{|c|}{ Number of needle passes } & & $0.386^{b}$ \\
\hline One & 273 (26.9) & $17(22.4)$ & \\
\hline Two or more & $741(73.1)$ & $59(77.6)$ & \\
\hline Needle angle & & & $0.392^{b}$ \\
\hline$<90^{\circ}$ & $611(60.3)$ & $42(55.3)$ & \\
\hline$\geq 90^{\circ}$ & $403(39.7)$ & $34(44.7)$ & \\
\hline Location of biopsy & & & $0.016^{b}$ \\
\hline Anterior chest wall & $356(35.1)$ & $17(22.4)$ & \\
\hline Lateral chest wall & $214(21.1)$ & $13(17.1)$ & \\
\hline Posterior chest wall & $444(43.8)$ & $46(60.5)$ & \\
\hline Pathological results & & & $<0.001^{\mathrm{b}}$ \\
\hline $\begin{array}{l}\text { Lung } \\
\text { adenocarcinoma }\end{array}$ & $392(38.7)$ & $31(40.8)$ & \\
\hline Lung metastases & $23(2.3)$ & $8(10.5)$ & \\
\hline Other tumors & $211(20.8)$ & $8(10.5)$ & \\
\hline $\begin{array}{l}\text { Inflammatory } \\
\text { changes }\end{array}$ & $190(18.7)$ & $11(14.5)$ & \\
\hline Other non-tumors & $151(14.9)$ & $8(10.5)$ & \\
\hline Non-diagnostic & $47(4.6)$ & $10(13.2)$ & \\
\hline
\end{tabular}

Higher-grade pulmonary hemorrhage was significantly more likely to occur with lung metastasis lesions compared with non-lung metastasis lesions $(\mathrm{P}=0.001)$. Continuous variables are expressed as mean \pm SD. Categorical data are No. (\%) of patients undergoing percutaneous CT-guided transthoracic lung biopsy. ${ }^{a}$, Student's $t$-test; ${ }^{b}$, Chi-square test. mPAD, main pulmonary artery diameter; $A A D$, ascending aorta diameter.

\section{Discussion}

In this retrospective study, we evaluated the risk factors for pulmonary hemorrhage and hemoptysis following PCTLB with a core needle and investigated whether mPAD/AAD ratio is a potential risk factor. The incidence of PCTLBrelated pulmonary hemorrhage $(31.38 \%)$ was consistent 
Table 3 Results of univariate analysis to determine risk factors for hemoptysis following percutaneous CT-guided transthoracic lung biopsy

\begin{tabular}{|c|c|c|c|}
\hline Variable & $\begin{array}{c}\text { No hemoptysis } \\
\quad(n=1,057)\end{array}$ & $\begin{array}{l}\text { Hemoptysis } \\
\quad(n=33)\end{array}$ & $P$ value \\
\hline Age (y) & $58.0 \pm 12.8$ & $56.5 \pm 14.9$ & $0.520^{a}$ \\
\hline Sex & & & $0.117^{\mathrm{b}}$ \\
\hline Male & $743(70.3)$ & $19(57.6)$ & \\
\hline Female & $314(29.7)$ & $14(42.4)$ & \\
\hline $\begin{array}{l}\text { History of chronic } \\
\text { obstructive pulmonary } \\
\text { disease }\end{array}$ & $67(6.3)$ & $2(6.1)$ & $1^{\mathrm{c}}$ \\
\hline History of hypertension & $198(18.7)$ & $12(36.4)$ & $0.011^{\mathrm{b}}$ \\
\hline $\begin{array}{l}\text { History of diabetes } \\
\text { mellitus }\end{array}$ & $128(12.1)$ & $2(6.1)$ & $0.416^{\mathrm{c}}$ \\
\hline Prior malignancy & $64(6.1)$ & $2(11.8)$ & $1^{\mathrm{c}}$ \\
\hline mPAD & & & $0.106^{\mathrm{b}}$ \\
\hline$<2.95 \mathrm{~cm}$ & $554(52.4)$ & $22(66.7)$ & \\
\hline$\geq 2.95 \mathrm{~cm}$ & $503(47.6)$ & $11(33.3)$ & \\
\hline $\mathrm{AAD}(\mathrm{cm})$ & $3.39 \pm 0.44$ & $3.36 \pm 0.54$ & $0.760^{\mathrm{b}}$ \\
\hline mPAD/AAD ratio & & & $0.716^{\mathrm{b}}$ \\
\hline$\leq 1$ & $871(82.4)$ & $28(84.8)$ & \\
\hline$>1$ & $186(17.6)$ & $5(15.2)$ & \\
\hline Lesion location & & & $0.533^{b}$ \\
\hline $\begin{array}{l}\text { Upper and middle } \\
\text { lobes }\end{array}$ & $648(61.3)$ & $22(66.7)$ & \\
\hline $\begin{array}{l}\text { Lower lobe and lung } \\
\text { hilar }\end{array}$ & $409(38.7)$ & $11(33.3)$ & \\
\hline Lesion characteristics & & & $0.572^{b}$ \\
\hline Solid & $847(80.1)$ & $25(75.8)$ & \\
\hline Subsolid & $87(8.2)$ & $4(12.1)$ & \\
\hline $\begin{array}{l}\text { Consolidation or } \\
\text { cavitary }\end{array}$ & $123(11.6)$ & $4(12.1)$ & \\
\hline Lesion size & & & $0.203^{b}$ \\
\hline$\leq 3 \mathrm{~cm}$ & $367(34.7)$ & $15(45.5)$ & \\
\hline$>3 \mathrm{~cm}$ & $690(65.3)$ & $18(54.5)$ & \\
\hline Lesion depth & & & $0.026^{\mathrm{b}}$ \\
\hline$\leq 3 \mathrm{~cm}$ & $193(18.3)$ & $4(12.1)$ & \\
\hline $3-5 \mathrm{~cm}$ & $378(35.8)$ & $6(18.2)$ & \\
\hline$>5 \mathrm{~cm}$ & $486(46)$ & $23(69.7)$ & \\
\hline Emphysema & $260(24.6)$ & $6(18.2)$ & $0.398^{\mathrm{b}}$ \\
\hline
\end{tabular}

Table 3 (continued)
Table 3 (continued)

\begin{tabular}{|c|c|c|c|}
\hline Variable & $\begin{array}{l}\text { No hemoptysis } \\
\qquad(n=1,057)\end{array}$ & $\begin{array}{l}\text { Hemoptysis } \\
\qquad(n=33)\end{array}$ & $P$ value \\
\hline $\begin{array}{l}\text { Number of needle } \\
\text { passes }\end{array}$ & & & $0.141^{\mathrm{b}}$ \\
\hline One & 210 (19.9) & $10(30.3)$ & \\
\hline Two or more & $847(80.1)$ & $23(69.7)$ & \\
\hline Needle angle & & & $0.657^{\mathrm{b}}$ \\
\hline$<90^{\circ}$ & $632(59.8)$ & $21(63.6)$ & \\
\hline$\geq 90^{\circ}$ & 425 (40.2) & $12(36.4)$ & \\
\hline Location of biopsy & & & $0.672^{b}$ \\
\hline Anterior chest wall & $360(34.1)$ & $13(39.4)$ & \\
\hline Lateral chest wall & $222(21.0)$ & $5(15.2)$ & \\
\hline Posterior chest wall & $475(44.9)$ & $15(45.5)$ & \\
\hline Pathological results & & & $0.542^{b}$ \\
\hline Lung adenocarcinoma & $410(38.8)$ & $13(39.4)$ & \\
\hline Other tumors & $244(23.1)$ & $6(18.2)$ & \\
\hline Inflammatory changes & $192(18.2)$ & $9(27.3)$ & \\
\hline Other non-tumor & $211(20.0)$ & $5(15.2)$ & \\
\hline Pulmonary hemorrhage & $320(30.3)$ & $22(66.7)$ & $<0.001^{\mathrm{b}}$ \\
\hline $\begin{array}{l}\text { Lower-grade pulmonary } \\
\text { hemorrhage }\end{array}$ & $254(24)$ & $12(36.4)$ & $0.104^{b}$ \\
\hline $\begin{array}{l}\text { Higher-grade pulmonary } \\
\text { hemorrhage }\end{array}$ & $66(6.2)$ & $10(30.3)$ & $<0.001^{b}$ \\
\hline \multicolumn{4}{|c|}{$\begin{array}{l}\text { Continuous variables are expressed as mean } \pm \text { SD. Categorical } \\
\text { data are No. (\%) of patients undergoing percutaneous CT- } \\
\text { guided transthoracic lung biopsy. a, Student's } t \text {-test; }{ }^{\text {b }} \text {, Chi- } \\
\text { square test; ' } \text {, Fisher's exact test. mPAD, main pulmonary artery } \\
\text { diameter; AAD, ascending aorta diameter. }\end{array}$} \\
\hline
\end{tabular}

with previously reported results (range, 30-65.6\%) (4,7,22). Moreover, the frequency of hemoptysis in our study was $3.03 \%$, which is similar to the rates in previous studies (range, $1.5-9.5 \%)(4,22,23,29)$. Our mortality rate of $0.09 \%$ $(1 / 1,090)$, which may be attributable to the risk of death from hemoptysis, was also comparable to the rate of $0.07 \%$ found in a large national study (6).

It is not recommended that patients with $\mathrm{PH}$ undergo lung biopsy $(9,10)$. Right-sided heart catheterization (RHC) is the gold standard in diagnosing $\mathrm{PH}$, but the invasive procedure is associated with risks and complications (30). Noninvasive approaches such as Doppler echocardiography is recommended for screening $\mathrm{PH}$ at the initial stage (31), 
Table 4 Results of multivariate logistic regression analyses for pulmonary hemorrhage, higher-grade pulmonary hemorrhage, and hemoptysis

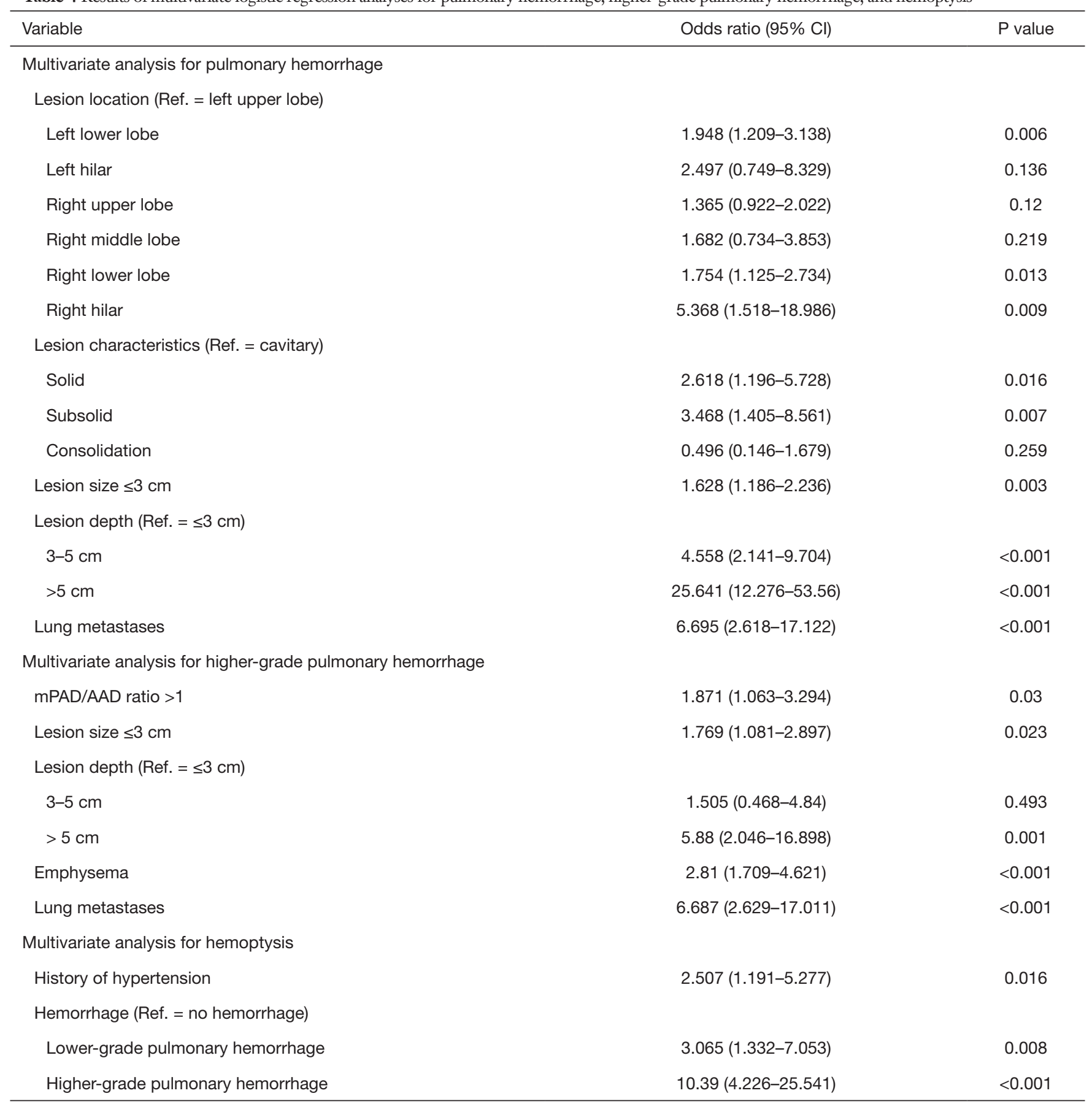

$\mathrm{Cl}$, confidence interval; Ref. Reference; mPAD, main pulmonary artery diameter; AAD, ascending aorta diameter.

but are usually not available when planning a biopsy in practice. Thus, $\mathrm{mPAD}$ or $\mathrm{mPAD} / \mathrm{AAD}$ ratio may be used as a surrogate for preliminary assessment of mean pulmonary artery pressure, as they are useful parameters for predicting $\mathrm{PH}$, and are highly specific but relatively insensitive
$(12,19)$. Additionally, mPAD/AAD ratio appears to be a better predictor of mean pulmonary artery pressure than mPAD alone, the ratio of mPAD over body surface area, or segmental arterial diameter $(14,15,32)$. Another more precise method to measure the main pulmonary artery, 
cross-sectional area of the main pulmonary artery, did not strengthen correlations with $\mathrm{PH}$ compared with the mPAD/AAD ratio (14). Previous studies found that a CTdemonstrated mPAD/AAD ratio $>1$ was associated with $\mathrm{PH}$, with a positive predictive value of more than $95 \%$ and a specificity of more than $90 \%(15,19,33)$. Moreover, the $\mathrm{mPAD} / \mathrm{AAD}$ ratio allows adjustment for anthropometric differences between patients to a greater extent than $\mathrm{mPAD}$ alone and can eliminate the influence of other factors on the pulmonary artery or aorta diameter (34). In our study, mPAD/AAD ratio $>1$ was not a significant risk factor for overall pulmonary hemorrhage and hemoptysis. However, a ratio $>1$ was a significant risk factor for higher-grade pulmonary hemorrhage. Hemorrhagic complications in patients with $\mathrm{PH}$ generally occur as a result of high perfusion pressure in the pulmonary artery; injury to pulmonary vessels during the biopsy procedure increases the risk of severe pulmonary hemorrhage and hemoptysis, as evidenced by the observation that an increase in $\mathrm{MPAD}$ was associated with severe hemoptysis following PCTLB (23). Thus, although PH may not increase the overall incidence of pulmonary hemorrhage and hemoptysis, once pulmonary bleeding occurs, it can exacerbate intrapulmonary hemorrhage. As demonstrated by our own and previous studies, the mPAD/AAD ratio appears to slightly outperform or show performance similar to established risk factors for higher-grade pulmonary hemorrhage, including smaller lesions, lesion location, lesion characteristics, sex, and age $(22,35)$. Consistent with previous reports $(22,23)$, in this study, mPAD enlargement was not a significant risk factor for overall pulmonary hemorrhage, higher-grade pulmonary hemorrhage, or hemoptysis. The mPAD/AAD ratio can be a clue to explaining the elevated pulmonary artery pressures and is easily measured on CT images. In our experience, an mPAD/AAD ratio $>1$ may increase the risk of the higher-grade pulmonary hemorrhage, and these cases require close post-PCTLB monitoring.

Other PCTLB-related variables were found to be independent predictors of pulmonary hemorrhage. The location of the lesion on the left or in the right lower lobe or right hilum was a risk factor for overall pulmonary hemorrhage. A possible explanation for this is that the lower lobes of the lung have a large capacity and can easily cause further tearing and enlargement of the biopsy orifice during the operation, while the lung hilum is far away from the chest wall at a deep location close to large blood vessels of the heart. This thereby increases the risk of injury to pulmonary vessels during the procedure. Basal lesions (36), along with subsolid lesions which may not be sufficiently compact to limit hemorrhage, have been reported as significant risk factors for pulmonary hemorrhage after PCTLB $(22,23,37)$. Small and deep lesions, as previously described in the literature, are independent risk factors for both overall and higher-grade pulmonary hemorrhage, $(4,22,38)$. We speculate that smaller lesions move more readily during respiration which could alter the direction of the needle, damaging the surrounding small vessels. Moreover, the needle path through the lung parenchyma is longer for deeper lesions, which increases the possibility of damage to the pleura and pulmonary vasculature.

We observed that emphysema around the lesion was a significant risk factor for overall and higher-grade pulmonary hemorrhage. A previous study found that imaging signs of emphysema were often associated with $\mathrm{PH}$ and increased the risk of pulmonary hemorrhage (39). Emphysema can also cause expansion of the bleeding space, which is linked to a reduced capacity to limit hemorrhage (22). Lung metastases were associated with both overall and higher-grade pulmonary hemorrhage, as metastatic lesions are probably generally small, or some metastases have a rich blood supply. Although PCTLB is a useful technique for diagnosing suspected pulmonary metastases as it allows open biopsy to be avoided in most cases (40), more attention must be paid to bleeding risk.

An interesting finding of our study was that a history of hypertension was a risk factor for hemoptysis, although it may be an aggravating factor rather than a cause. Recurrent hemoptysis has been reported in patients with a history of hypertension after flexible bronchoscopy (41). Hemoptysis following PCTLB is often accompanied by pulmonary hemorrhage; therefore, pulmonary hemorrhage during or after the procedure must be monitored to determine whether patients also have hemoptysis. Furthermore, in female patients, subsolid nodules and deeply located lesions were found to be independent risk factors for hemoptysis $(23,29)$, although this was not substantiated in the present study, possibly due to the small number of hemoptysis cases.

There were some limitations to this study. Firstly, the retrospective design introduced an unavoidable bias as patients were preselected to undergo CT-guided lung biopsy. Secondly, we were unable to confirm the predictive value of the $\mathrm{mPAD}$ and $\mathrm{mPAD} / \mathrm{AAD}$ ratio for $\mathrm{PH}$ in our study, as we could not collect data on pulmonary artery pressure measured directly by RHC or echocardiography owing to the small number of cases. Thus, very few patients have a clear history of $\mathrm{PH}$, and the clinician may have 
declined performing biopsy on those patients with severe pulmonary hypertension. Therefore, there is a limitation to the statistical power of the analyses. We hope that future prospective studies are designed to assess the relationship between $\mathrm{PH}$ and the risk of hemorrhagic complications. Thirdly, the diagnosis of pulmonary hemorrhage was screened from CT images during or immediately after the biopsy. It is thus possible that delayed pulmonary hemorrhage related to the procedure may have been missed before or after hospital discharge. Moreover, a small amount of pulmonary hemorrhage is difficult to detect, especially in cases of subpleural or consolidation lesions, and hemoptysis that developed after the biopsy was not recorded in the EMR, which could have led to the underestimation of the incidence of postoperative bleeding. Finally, other variables (e.g., operator skill, biopsy type, or needle gauge) that are difficult to assess were not considered in the analysis.

In conclusion, we showed that CT-detected pulmonary artery enlargement (mPAD/AAD ratio $>1$ ), a small and deeply located lesion, the presence of emphysema around the lesions, and suspected lung metastases are significant risk factors for PCTLB-related higher-grade pulmonary hemorrhage. The mPAD/AAD ratio is especially useful in clinical practice, given that it can be determined with minimal training and is reproducible and routinely available when measured at the pulmonary artery bifurcation. Risk factors for hemoptysis were a history of hypertension and the occurrence of pulmonary hemorrhage, especially of a higher grade. Our findings provide a tool for the timely identification of patients undergoing PCTLB who are at risk of hemorrhage and hemoptysis so that appropriate interventions can be implemented, and postoperative outcomes improved.

\section{Acknowledgments}

Funding: None.

\section{Footnote}

Conflicts of Interest: All authors have completed the ICMJE uniform disclosure form (available at http://dx.doi. org/10.21037/qims-19-1024). The authors have no conflicts of interest to declare.

Ethical Statement: The study protocol was approved by our institutional review board (2019-KY-056-01) with a waiver of informed consent obtained from each patient.
Open Access Statement: This is an Open Access article distributed in accordance with the Creative Commons Attribution-NonCommercial-NoDerivs 4.0 International License (CC BY-NC-ND 4.0), which permits the noncommercial replication and distribution of the article with the strict proviso that no changes or edits are made and the original work is properly cited (including links to both the formal publication through the relevant DOI and the license). See: https://creativecommons.org/licenses/by-nc-nd/4.0/.

\section{References}

1. Travis WD, Brambilla E, Noguchi M, Nicholson AG, Geisinger K, Yatabe Y, Powell CA, Beer D, Riely G, Garg K, Austin JH, Rusch VW, Hirsch FR, Jett J, Yang PC, Gould M; American Thoracic Society. International Association for the Study of Lung Cancer/American Thoracic Society/European Respiratory Society: International multidisciplinary classification of lung adenocarcinoma: Executive summary. Proc Am Thorac Soc 2011;8:381-85.

2. Khouri NF, Stitik FP, Erozan YS, Gupta PK, Kim WS, Scott WJ, Hamper UM, Mann RB, Eggleston JC, Baker RR. Transthoracic needle aspiration biopsy of benign and malignant lung lesions. AJR Am J Roentgenol 1985;144:281-88.

3. Wu CC, Maher MM, Shepard JA. Complications of CT-guided percutaneous needle biopsy of the chest: Prevention and management. AJR Am J Roentgenol 2011;196:W678-82.

4. Yeow KM, Su IH, Pan KT, Tsay PK, Lui KW, Cheung YC, Chou AS. Risk factors of pneumothorax and bleeding: Multivariate analysis of 660 CT-guided coaxial cutting needle lung biopsies. Chest 2004;126:748-54.

5. Wiener RS, Wiener DC, Gould MK. Risks of transthoracic needle biopsy: How high? Clin Pulm Med 2013;20:29-35.

6. Tomiyama N, Yasuhara Y, Nakajima Y, Adachi S, Arai Y, Kusumoto M, Eguchi K, Kuriyama K, Sakai F, Noguchi M, Murata K, Murayama S, Mochizuki T, Mori K, Yamada K. CT-guided needle biopsy of lung lesions: A survey of severe complication based on 9783 biopsies in Japan. Eur J Radiol 2006;59:60-4.

7. Heerink WJ, de Bock, GH, de Jonge GJ, Groen HJ, Vliegenthart R, Oudkerk M. Complication rates of CTguided transthoracic lung biopsy: Meta-analysis. Eur Radiol 2017;27:138-48.

8. Westcott JL, Rao N, Colley DP. Transthoracic 
needle biopsy of small pulmonary nodules. Radiology 1997;202:97-103.

9. Manhire A, Charig M, Clelland C, Gleeson F, Miller R, Moss H, Pointon K, Richardson C, Sawicka E. Guidelines for radiologically guided lung biopsy. Thorax 2003;58:920-36.

10. Sargent EN, Turner AF, Gordonson J, Schwinn CP, Pashky O. Percutaneous pulmonary needle biopsy. Report of 350 patients. Am J Roentgenol Radium Ther Nucl Med 1974;122:758-68.

11. Corson N, Armato SG, Labby ZE, Straus C, Starkey A, Gomberg-Maitland M. CT-based pulmonary artery measurements for the assessment of pulmonary hypertension. Acad Radiol 2014;21:523-30.

12. Mahammedi A, Oshmyansky A, Hassoun PM, Thiemann DR, Siegelman SS. Pulmonary artery measurements in pulmonary hypertension: The role of computed tomography. J Thorac Imaging 2013;28:96-103.

13. Kam JC, Doraiswamy V, Elnahar Y, Abdul-Jawad S, Debari VA, Klukowicz AJ, Shamoon F, Miller RA. CT scanning in the evaluation of pulmonary hypertension. Lung 2013;191:321-26.

14. Devaraj A, Wells AU, Meister MG, Corte TJ, Wort SJ, Hansell DM. Detection of pulmonary hypertension with multidetector CT and echocardiography alone and in combination. Radiology 2010;254:609-16.

15. Ascha M, Renapurkar RD, Tonelli AR. A review of imaging modalities in pulmonary hypertension. Ann Thorac Med 2017;12:61-73.

16. Shin S, King CS, Brown AW, Albano MC, Atkins M, Sheridan MJ, Ahmad S, Newton KM, Weir N, Shlobin OA. Pulmonary artery size as a predictor of pulmonary hypertension and outcomes in patients with chronic obstructive pulmonary disease. Respir Med 2014;108:1626-32.

17. Boerrigter B, Mauritz GJ, Marcus JT, Helderman F, Postmus PE, Westerhof N, Vonk-Noordegraaf A. Progressive dilatation of the main pulmonary artery is a characteristic of pulmonary arterial hypertension and is not related to changes in pressure. Chest 2010;138:1395-401.

18. Altschul E, Remy-Jardin M, Machnicki S, Sulica R, Moore JA, Singh A, Raoof S. Imaging of pulmonary hypertension: Pictorial essay. Chest 2019;156:211-27.

19. Ng CS, Wells AU, Padley, SP. A CT sign of chronic pulmonary arterial hypertension: The ratio of main pulmonary artery to aortic diameter. J Thorac Imaging 1999;14:270-8.
20. Iyer AS, Wells JM, Vishin S, Bhatt SP, Wille KM, Dransfield MT. CT Scan-Measured Pulmonary Artery to Aorta Ratio and Echocardiography for Detecting Pulmonary Hypertension in Severe COPD. Chest 2014;145:824-32.

21. Barr RG, Bluemke DA, Ahmed FS, Carr JJ, Enright PL, Hoffman EA. Jiang R, Kawut SM, Kronmal RA, Lima JOAC. Percent Emphysema, Airflow Obstruction, and Impaired Left Ventricular Filling. N Engl J Med 2010;362:217-27.

22. Tai R, Dunne RM, Trotman-Dickenson B, Jacobson FL, Madan R, Kumamaru KK, Hunsaker AR. Frequency and severity of pulmonary hemorrhage in patients undergoing percutaneous CT-guided transthoracic lung biopsy: Single-institution experience of 1175 cases. Radiology 2016;279:287-96.

23. Hwang EJ, Park CM, Yoon SH, Lim HJ, Goo JM. Risk factors for haemoptysis after percutaneous transthoracic needle biopsies in 4,172 cases: Focusing on the effects of enlarged main pulmonary artery diameter. Eur Radiol 2018;28:1410-9.

24. Digumarthy SR, Kovacina B, Otrakji A, Lanuti M, Shepard JA, Sharma A. Percutaneous CT guided lung biopsy in patients with pulmonary hypertension: Assessment of complications. Eur J Radiol 2016;85:466-71.

25. Corder R. Hemoptysis. Emerg Med Clin North Am 2003;21:421-35.

26. Wells JM, Washko GR, Han MK, Abbas N, Nath H, Mamary AJ, Regan E, Bailey WC, Martinez FJ, Westfall E, Beaty TH, Curran-Everett D, Curtis JL, Hokanson JE, Lynch DA, Make BJ, Crapo JD, Silverman EK, Bowler RP, Dransfield MT;COPDGene Investigators;ECLIPSE Study Investigators. Pulmonary arterial enlargement and acute exacerbations of COPD. N Engl J Med 2012;367:913-21.

27. Grubstein A, Benjaminov O, Dayan DB, Shitrit D, Cohen M, Kramer MR. Computed tomography angiography in pulmonary hypertension. Isr Med Assoc J 2008;10:117-20.

28. Lim W, Ridge CA, Nicholson AG, Mirsadraee S. The 8th lung cancer TNM classification and clinical staging system: review of the changes and clinical implications. Quant Imaging Med Surg 2018;8:709-18.

29. Song YS, Park CM, Park KW, Kim KG, Lee HJ, Shim MS, Goo JM. Does antiplatelet therapy increase the risk of hemoptysis during percutaneous transthoracic needle biopsy of a pulmonary lesion? AJR Am J Roentgenol 2013;200:1014-9.

30. Badesch DB, Champion HC, Sanchez MA, Hoeper MM, Loyd JE, Manes A, McGoon M, Naeije R, Olschewski 
H, Oudiz RJ, Torbicki A. Diagnosis and assessment of pulmonary arterial hypertension. J Am Coll Cardiol 2009;54:S55-66.

31. Barst RJ, McGoon M, Torbicki A, Sitbon O, Krowka MJ, Olschewski H, Gaine S. Diagnosis and differential assessment of pulmonary arterial hypertension. J Am Coll Cardiol 2004;43:40S-47S.

32. Murray TI, Boxt LM, Katz J, Reagan K, Barst RJ. Estimation of Pulmonary Artery Pressure in Patients with Primary Pulmonary Hypertension by Quantitative Analysis of Magnetic Resonance Images. Journal of Thoracic Imaging 1994;9:198-204.

33. Grosse C, Grosse A. CT findings in diseases associated with pulmonary hypertension: a current review. Radiographics 2010;30:1753-77.

34. Han MK, Kazerooni EA, Lynch DA, Liu LX, Murray S, Curtis JL, Criner GJ, Kim V, Bowler RP, Hanania NA, Anzueto AR, Make BJ, Hokanson JE, Crapo JD, Silverman EK, Martinez FJ, Washko GR;COPDGene Investigators. Chronic obstructive pulmonary disease exacerbations in the COPDGene study: Associated radiologic phenotypes. Radiology 2011;261:274-82.

35. Fontaine-Delaruelle C, Souquet PJ, Gamondes D, Pradat E, de Leusse A, Ferretti GR, Couraud S. Predictive factors of complications during CT-guided transthoracic biopsy. Rev Pneumol Clin 2017;73:61-7.

Cite this article as: Zhu J, Qu Y, Wang X, Jiang C, Mo J, Xi J, Wen Z. Risk factors associated with pulmonary hemorrhage and hemoptysis following percutaneous CT-guided transthoracic lung core needle biopsy: a retrospective study of 1,090 cases. Quant Imaging Med Surg 2020;10(5):1008-1020. doi: 10.21037/ qims-19-1024
36. Nour-Eldin NEA, Alsubhi M, Naguib NN, Lehnert T, Emam A, Beeres M, Bodelle B, Koitka K, Vogl TJ, Jacobi $\mathrm{V}$. Risk factor analysis of pulmonary hemorrhage complicating CT-guided lung biopsy in coaxial and noncoaxial core biopsy techniques in 650 patients. Eur J Radiol 2014;83:1945-52.

37. Lee SM, Park CM, Lee KH, Bahn YE, Kim JI, Goo JM. C-arm cone-beam CT-guided percutaneous transthoracic needle biopsy of lung nodules: Clinical experience in 1108 patients. Radiology 2014;271:291-300.

38. Yildirim E, Kirbas I, Harman A, Ozyer U, Tore HG, Aytekin C, Boyvat F. CT-guided cutting needle lung biopsy using modified coaxial technique: Factors effecting risk of complications. Eur J Radiol 2009;70:57-60.

39. Heyer CM, Reichelt S, Peters SA, Walther JW, Müller $\mathrm{K}$, Nicolas, V. Computed tomography-navigated transthoracic core biopsy of pulmonary lesions. Acad Radiol. 2008;15:1017-26.

40. Galluzzo A, Genova C, Dioguardi S, Midiri M, Cajozzo M. Current role of computed tomography-guided transthoracic needle biopsy of metastatic lung lesions. Future Oncol 2015;11:43-6.

41. Lee MK, Kim SH, Yong SJ, Shin KC, Kim HS, Yu TS, Choi EH, Lee WY. Moderate hemoptysis: Recurrent hemoptysis and mortality according to bronchial artery embolization. Clin Respir J 2015;9:53-64. 